Revista de

Contabilidade e

Organizações

www.rco.usp.br
DOI: http://dx.doi.org/10.11606/rco.v11i29.122331
Journal of

Accounting and

Organizations

\title{
Fatores determinantes das book-tax differences
}

\author{
Kellma Bianca Cardoso Fonseca ${ }^{a}$; Patricia de Souza Costa ${ }^{a}$
}

${ }^{a}$ Universidade Federal de Uberlândia

\section{Informações do Artigo}

Histórico do Artigo

Recebido: 26 de outubro de 2016

Aceito: 22 de março de 2017

Palavras-chave:

Book-Tax Differences.

Fatores Institucionais.

Fatores não Institucionais.

\begin{abstract}
Resumo
Este artigo tem por objetivo identificar quais são os fatores institucionais e não institucionais que determinam as book-tax differences (BTD) das companhias abertas brasileiras. A amostra da pesquisa é composta por 124 companhias, com dados disponíveis para o período de 2010 a 2015. Os resultados indicam que a BTD total apresenta relação positiva com as variáveis rentabilidade, liquidez e a BTD defasada. Quando desmembrados os tipos de BTD, a rentabilidade e a BTD defasada estão positivamente associadas à BTD temporária. A rentabilidade e a liquidez estão positivamente associadas à BTD permanente. Portanto, os resultados sugerem que a rentabilidade está associada com todos os tipos de BTD (total, temporária e permanente), ou seja, as empresas mais rentáveis evidenciam maior diferença entre o lucro contábil e o lucro tributável. Além disso, os resultados sugerem que a segregação da BTD por tipos (temporária e permanente) é relevante na análise dos determinantes dessa variável.
\end{abstract}

Copyright $\odot 2017$ FEA-RP/USP. Todos os direitos reservados

\section{INTRODUÇÃO}

As diferenças entre o lucro contábil e o lucro tributável são denominadas de book-tax differences (BTD) (Ferreira, Matinez, Costa, \& Passamani, 2012). O lucro contábil é apurado de acordo com as normas societárias, que permitem escolhas contábeis e a aplicação do subjetivismo responsável, o que pode tornar a apuração desse lucro mais subjetiva (Ferreira et al., 2012). Por outro lado, o lucro tributável é apurado de acordo com normas fiscais objetivas e rígidas, para fins de apuração do imposto de renda (Niyama \& Silva, 2011). Dessas diferenças entre as normas, primeiramente, origina-se a BTD.

A BTD também pode ser provocada pelo gerenciamento de resultados e pelo gerenciamento tributário (Desai, 2005). O gestor, com o intuito de maximizar os seus interesses, está motivado em adotar políticas contábeis que possibilitem aumentar o lucro contábil com o objetivo de atrair investidores, e, por outro lado, para escolher políticas fiscais que minimizem o lucro tributável, a fim de evitar os impostos (Long, Ye, \& Lv, 2013). Para esses autores, tanto o gerenciamento de resultados contábeis quanto o de resultados fiscais podem conduzir à ampliação da BTD de maneira anormal. Assim, a BTD pode ser produto tanto de fatores institucionais (BTD normal) quanto de fatores não-institucionais (BTD anormal) (Manzon \& Plesko, 2002, Long et al., 2013, Kouba \& Anis, 2015).

Long et al. (2013) investigaram a relação entre os fatores não-institucionais e a BTD, utilizando uma amostra de companhias abertas chinesas. Esses autores encontraram uma relação negativa entre a BTD e os seguintes fatores: relação entre o valor de mercado e o lucro, relação entre o valor de mercado e o patrimônio líquido, liquidez, alavancagem e a taxa de crescimento do lucro por ação. Há uma relação positiva entre a BTD e a taxa de investidores institucionais e o tamanho da empresa. Koubaa e Anis (2015) verificaram a relação entre fatores institucionais (lucratividade, crescimento das vendas e crescimento de imobilizado) e não institucionais (accruals, relação entre valor de mercado e patrimonial, liquidez, alavancagem, taxa de ações institucionais e BTD defasada) e a BTD, valendo-se da amostra de vinte e oito companhias tunisianas com dados do período de 2005 a 2012. Os resultados sugerem que a lucratividade, o crescimento das vendas, os accruals, a alavancagem e a relação entre o

Autor Correspondente: Tel (34) 3239-4411

E-mail: kell_bianca1994@hotmail.com (K. B. C. Fonseca); patriciacosta_1@yahoo.com.br (P. S. Costa)

Universidade Federal de Uberlândia - Av. João Naves de Ávila, 2121 - Santa Mônica, Uberlândia - MG, 38408-100 
valor de mercado e valor contábil estão relacionadas com a BTD.

Manzon e Plesko (2002) e Long et al. (2013) ressaltam que são escassas, na literatura acadêmica, pesquisas que busquem identificar os fatores que determinam a BTD. Long et al. (2013) investigaram os fatores determinantes da BTD utilizando uma amostra de companhias da China, país de sistema legal misto (code law e common law), enquanto Koubaa e Anis (2015) analisaram companhias da Tunísia, país de origem common law. A conjuntura do Brasil país de origem code law e conhecido pelo alto nível de vinculação entre a contabilidade societária e a fiscal pode afetar o comportamento da BTD (Marques, Costa, \& Silva, 2016) e a relação dos fatores determinantes dessa diferença.

Além disso, Long et al. (2013) e Koubaa e Anis (2015) analisaram apenas a BTD total. Wahab e Holland (2014), Costa e Lopes (2015) e Marques et al. (2016) e Santos, Costa e Silva (2016) asseveram que a análise da BTD por tipos (permanente e temporária) pode trazer informações adicionais sobre a vinculação entre a contabilidade financeira e a fiscal. A BTD temporária pode estar mais associada com o gerenciamento de resultados do que a BTD permanente (Martinez \& Passamani, 2014). A BTD permanente pode trazer informações relevantes sobre as atividades de evasão fiscal (Wilson, 2009) e gestão tributária estratégica (Frank, Lynch, \& Rego, 2009).

Nesse sentido, considerando a escassez de pesquisas sobre o tema desta pesquisa, o problema de pesquisa é: quais os fatores determinantes dos tipos de book-tax differences no Brasil? O objetivo é identificar quais são os fatores institucionais e não institucionais que determinam os tipos de book-tax differences das companhias abertas brasileiras. A amostra da pesquisa é composta por 124 companhias abertas brasileiras com dados disponíveis para o período de 2010 a 2015.

Os resultados deste estudo podem contribuir para a literatura sobre BTD, uma vez que a análise dos determinantes que influenciam a BTD é considerada recente e inovadora (Koubaa \& Anis, 2015). Koubaa e Anis (2015) ressaltam que são poucos os estudos sobre o tema desta pesquisa. No Brasil, não foram encontradas pesquisas com o objetivo de identificar os determinantes da BTD em companhias abertas. A maioria dos estudos é realizada com amostras de empresas de países common law e com foco apenas na BTD total. Este estudo avança utilizando uma amostra de companhias brasileiras, país de origem code law, e analisando a BTD total, a permanente e a temporária. A BTD temporária pode estar mais relacionada ao gerenciamento de resultados, enquanto a BTD permanente pode estar vinculada ao gerenciamento fiscal (Wilson, 2009, Costa \& Lopes, 2015). Além disso, os resultados desta pesquisa podem ser úteis para investidores, reguladores, analistas e normatizadores conhecerem o perfil das companhias abertas brasileiras quanto à vinculação entre a contabilidade societária e fiscal.

\section{REFERENCIAL TEÓRICO}

\subsection{Book-Tax Differences}

A diferença entre o lucro contábil e o lucro tributável denomina-se book-tax differences (BTD). Existem, pelo menos, três razões pelas quais o lucro contábil difere do lucro tributável. Primeiramente, por causa das diferenças entre as normas contábeis e as normas fiscais (Niyama \& Silva, 2011). O lucro contábil é apurado com base nos princípios contábeis geralmente aceitos (PCGA) e na representação fidedigna, podendo conter escolhas contábeis e subjetivismo, enquanto o cálculo do lucro tributável segue as regras fiscais, mais objetivas do que as normas societárias (Ferreira et al., 2012). As diferenças entre essas normas podem resultar na apuração de um lucro contábil diferente do lucro tributável, o que resulta na BTD (Ferreira, et al., 2012).

De acordo com Kvaal e Nobes (2013), alguns países propiciam maior desvinculação entre as normas contábeis e as normas fiscais, como por exemplo, os países common law, em que prevalece a essência econômica sobre a forma jurídica, baseiam-se em princípios e não em regas detalhadas, exibem maior BTD. Nos países code law, prevalece a forma jurídica sobre a essência econômica, e regras mais detalhadas exigem maior conformidade entre os dois sistemas, sendo, assim, menor a BTD (Niyama, 2005).

A segunda razão para a origem da BTD é o gerenciamento de resultados. A contabilidade societária permite critérios alternativos para registro, mensuração e/ou evidenciação de um evento econômico de forma objetiva e verificável. Os administradores podem manipular o lucro contábil para atender aos objetivos do mercado financeiro e influenciar a representação fidedigna da real situação da empresa (Nakao, 2012). Por outro lado, a contabilidade tributária reduz o número de critérios para a mensuração dos resultados tributários, exigindo maior 
objetividade no registro dos eventos econômicos. Dessa forma, esses resultados poderão ser distintos, surgindo, assim, a BTD (Marques et al., 2016).

A terceira razão para o surgimento da BTD é o gerenciamento tributário (Desai, 2005). O gerenciamento tributário ocorre quando os gestores manipulam as ambiguidades e incertezas das leis tributárias, com o objetivo de reduzir a carga tributária e amenizar o pagamento de impostos (Formigoni, Antunes, \& Paulo, 2009). Na maioria das vezes, essa prática é empregada para reduzir o lucro tributário (Formigoni et al., 2009).

A BTD pode ser classificada em dois tipos: permanente e temporária (Ferreira et al., 2012). As diferenças permanentes ocorrem quando um evento (despesa ou receita) é reconhecido contabilmente, mas não possui efeito tributário, sendo assim, não são reconhecidas no sistema tributário (Formigoni et al., 2009). As diferenças temporárias, por sua vez, ocorrem quando os sistemas contábeis e tributários reconhecem as mesmas receitas e despesas, mas divergem quanto ao momento do reconhecimento (Ferreira et al., 2012). Ou seja, em algum momento no futuro, a BTD temporária será anulada.

Tang (2006) afirma que quanto maior o gerenciamento de resultados registrado pela empresa, menor a qualidade das informações divulgadas nas demonstrações contábeis. De acordo com Tang e Firth (2011), em empresas chinesas, o gerenciamento de resultados pode explicar parte do montante da BTD. Hanlon (2005) identificou que empresas com maior BTD revelam uma baixa persistência dos lucros em relação às empresas que apresentam menor BTD, isso sugere que os altos valores de BTD estão relacionados à baixa qualidade de lucro.

\subsection{Fatores determinantes da book-tax differences e hipóteses de pesquisa}

As pesquisas que buscam identificar os fatores determinantes da BTD são recentes (Koubaa \& Anis, 2015). No Brasil, não foram encontradas pesquisas que analisam esse tema. Alguns estudos realizados nos Estados Unidos (por exemplo, Manzon \& Plesko, 2002, Mills, Newberry, \& Trautman, 2002, Plesko, 2004) verificaram um crescimento da BTD ao longo dos anos de 1990, e concluíram que esse crescimento não pode ser explicado, exclusivamente, por fatores institucionais, mas também por outros fatores não-institucionais. De acordo com Long, Ye e Lv (2013), as diferenças institucionais referem-se às BTDs que resultam das diferenças entre as normas contábeis e tributárias - enquanto as diferenças não institucionais surgem de outros fatores, como, por exemplo, o gerenciamento de resultados. Desse modo, os fatores determinantes da BTD podem ser classificados em institucionais (rentabilidade, crescimento das vendas, imobilizado, propriedade institucional) e não-institucionais (accruals, retorno das ações, liquidez, tamanho da empresa e alavancagem) (Koubaa, \& Anis, 2015).

Long et al. (2013) investigaram a relação entre fatores não-institucionais e a BTD, utilizando uma amostra de companhias abertas chinesas, no período de 2008 a 2010. Esses autores encontraram uma relação negativa entre a BTD e os seguintes fatores: relação entre o valor de mercado e o lucro, relação entre o valor de mercado e o patrimônio líquido, liquidez, alavancagem e a taxa de crescimento do lucro por ação. Encontrou-se também uma relação positiva entre a BTD e a taxa de investidores institucionais e o tamanho da empresa.

Por outro lado, Koubaa e Anis (2015) verificaram a relação da BTD entre fatores institucionais e não institucionais, utilizando uma amostra de vinte e oito companhias tunisianas com dados do período de 2005 a 2012. Os resultados sugerem uma relação significativa e positiva entre a BTD e as seguintes variáveis: lucratividade, o crescimento das vendas, a liquidez e a variável BTD defasada e também uma relação significativa e negativa entre a BTD e a alavancagem e o tamanho da empresa. Os resultados são importantes para reduzir o grau de assimetria da informação (Koubaa, \& Anis, 2015).

As diferenças de resultados entre as pesquisas de Long et al. (2013) e Koubaa e Anis (2015) podem estar relacionadas ao fato de que, em 2006, a China emitiu novas normas de contabilidade de acordo com a International Financial Report Standards (IFRS); a amostra de pesquisa foi retirada das empresas chinesas do setor de agricultura, silvicultura pecuária e da pesca, para as quais, de acordo com a nova legislação estabelecida, seria difícil calcular a BTD.

Percebe-se que não existe consenso entre os resultados encontrados por Long et al. (2013) e Koubaa e Anis (2015) quanto à relação entre os fatores institucionais e não institucionais e a BTD. Essa falta de consenso sugere a necessidade de mais pesquisas sobre o tema. Assim, as hipóteses que serão testadas nesta pesquisa e as principais bases teóricas para testá-las estão resumidas na Tabela 1. 
Tabela 1. Fatores determinantes da BTD

\begin{tabular}{|c|c|c|c|}
\hline & Fatores & Sinal Esperado & Autores \\
\hline \multirow{3}{*}{ Institucionais } & Rentabilidade & $(+)$ & $\begin{array}{l}\text { Manzon e Plesko (2002), Sodan (2012), Koubaa e } \\
\text { Anis (2015) }\end{array}$ \\
\hline & Crescimento das Vendas & $(+)$ & Manzon e Plesko (2002), Koubaa e Anis (2015) \\
\hline & Imobilizado & $(+)$ & Manzon e Plesko (2002) \\
\hline \multirow{3}{*}{$\begin{array}{l}\text { Não } \\
\text { institucionais }\end{array}$} & Accruals & $(+)$ & Koubaa e Anis(2015), Ferreira et al. (2012) \\
\hline & Retorno das ações & $(-)$ & Koubaa e Anis (2015) \\
\hline & Liquidez & $(+)$ & Costa e Lopes (2015), Koubaa e Anis (2015) \\
\hline \multirow{2}{*}{$\begin{array}{l}\text { Variáveis de } \\
\text { Controle }\end{array}$} & Tamanho da empresa & $(-)$ & Chan, Lin e Mo (2010), Koubaa e Anis (2015) \\
\hline & Alavancagem & $(+)$ & Koubaa e Anis (2015) \\
\hline
\end{tabular}

Fonte: Elaborado pelas autoras.

Empresas altamente rentáveis possuem relação positiva e significativa com a BTD, pois estas companhias podem ampliar de forma efetiva as deduções e créditos tributários para se beneficiarem das isenções fiscais e para reduzir os rendimentos tributáveis (Manzon, \& Plesko, 2002, Sodan, 2012, Koubaa, \& Anis, 2015). Os gestores usam os benefícios tributários numa tentativa de reduzir os tributos a serem pagos, ampliando, assim, as BTD (Sodan, 2012). Nesse contexto, tem-se a primeira hipótese de pesquisa:

$\mathbf{H}_{1}$ : A rentabilidade das companhias abertas brasileiras possui uma relação positiva com a BTD.

Manzon e Plesko (2002) afirmam que há uma correlação positiva entre crescimento das vendas e a BTD, pois, com o aumento das receitas, as companhias em desenvolvimento tendem a fazer gerenciamento de resultados para reduzir a carga tributária. De acordo com Koubaa e Anis (2015), umas das formas de reduzir as receitas de vendas e a classificação das perdas estimadas com créditos de liquidação duvidosa, que são imediatamente reconhecidas como perdas nas demonstrações financeiras, para a redução da receita anual - consequentemente uma redução no lucro contábil. Assim, a segunda hipótese de pesquisa é: leiras.

$\mathbf{H}_{2}$ : Existe uma relação positiva entre o crescimento das vendas e a BTD das companhias abertas brasi-

O crescimento do investimento no imobilizado tem relação positiva com a BTD, pois, para a contabilidade societária, há mais critérios para os gestores contabilizarem a depreciação, como, por exemplo, linear ou depreciação acelerada, diferente das normas fiscais em que a depreciação será de acordo com a legislação tributária (Manzon, \& Plesko, 2002, Costa, \& Lopes, 2015). De acordo com Mills e Newberry (2001), empresas com maiores investimentos no imobilizado têm maior incentivo para escolher práticas contábeis que aumentem o lucro contábil, assim, espera-se uma relação positiva entre a BTD e o imobilizado. Nesse cenário, surge a terceira hipótese de pesquisa:

$\mathbf{H}_{3}$ : Existe uma relação positiva entre o imobilizado e a BTD das companhias abertas brasileiras.

A BTD pode ser, parcialmente, explicada pelas práticas de gerenciamento empregadas pelas empresas que visam à gestão de lucros, nelas os gestores são, frequentemente, estimulados a aumentar os lucros contábeis, ao mesmo tempo em que visam reduzir a carga tributária (Koubaa, \& Anis, 2015). Sendo assim, essa manipulação pode levar ao aumento das BTD, sugerindo que os accruals têm relação positiva com a BTD (Koubaa, \& Anis, 2015), o que conduz à quarta hipótese de pesquisa.

$\mathbf{H}_{4}$ : Os accruals possuem uma relação positiva com a BTD das companhias abertas brasileiras.

O retorno das ações pode estar relacionado com o nível de BTD, pois quanto maior o nível das BTD menor o retorno das ações, uma vez que o maior volume de BTD pode sinalizar para os investidores maior possibilidade de gerenciamento de resultado (Tang, 2006). Os investidores utilizam-se das informações contidas na BTD para avaliar o futuro desempenho das empresas, ou seja, os investidores subestimam as expectativas e rendimentos futuros para a tomada de decisão (Hanlon, 2005). Nessa perspectiva, surge a quinta hipótese de pesquisa:

$\mathbf{H}_{5}$ : $\mathrm{O}$ retorno das ações tem relação negativa com a BTD das companhias abertas brasileiras. 
Costa e Lopes (2015) sugerem que a liquidez pode afetar positivamente a BTD, uma vez que empresas com baixa liquidez são mais propensas ao gerenciamento de resultados. Por outro lado, em empresas com elevadas receitas, os gestores buscam uma redução na base tributária para pagarem menores tributos (Koubaa, \& Anis, 2015). Portanto, tem-se a sexta hipótese de pesquisa:

$\mathbf{H}_{6}$ : A liquidez da empresa tem relação positiva com a BTD das companhias abertas brasileiras.

Frank et al. (2009) chegaram à conclusão de que as companhias com níveis relativamente altos de dívidas foram estimuladas a gerenciar os resultados para evitar a violação de contratos. Empresas com alta alavancagem financeira tendem a apresentar menor qualidade das informações nas demonstrações contábeis, ou seja, aumentam a prática de gerenciamento de resultados e, consequentemente, aumentam a BTD, o que sugere a sétima hipótese de pesquisa:

$\mathbf{H}_{7}$ : Existe uma relação positiva entre o nível de alavancagem e a BTD das companhias abertas brasileiras.

O ativo total tem sido empregado como proxy para medir o tamanho da empresa (Chan et al., 2010). Chan et al. (2010) identificaram uma relação negativa entre o tamanho da empresa e a BTD, argumentam que grandes empresas tendem a obedecer mais aos regulamentos fiscais, porque são mais fiscalizadas pelos órgãos reguladores. Assim, tem-se a oitava hipótese de pesquisa:

$\mathbf{H}_{\mathbf{8}}$ : Existe uma relação negativa entre o tamanho da empresa e a BTD das companhias abertas brasileiras.

De acordo com o exposto na Tabela 1, espera-se uma relação positiva entre a BTD e a a rentabilidade, o crescimento das vendas, o imobilizado, os accruals, a liquidez e a alavancagem. Por outro lado, espera-se uma relação negativa entre a BTD e o retorno das ações e o tamanho das empresas.

\section{METODOLOGIA}

A amostra inicial da pesquisa é composta por 352 companhias não financeiras ativas e listadas na BM\&FBovespa no período de 2009 a 2015 (Tabela 2). Foram excluídos os dados do ano de 2009, uma vez que esse período foi utilizado apenas para cálculo das variáveis defasadas. Também foram excluídas as companhias que não possuíam os dados para cálculo de todas as variáveis dos modelos especificados nas Equações 1 e 2. Assim, a amostra válida do estudo é de 124 companhias abertas brasileiras com dados para o período de 2010 a 2015.

Tabela 2. Composição da Amostra

\begin{tabular}{lcc}
\hline \multicolumn{1}{c}{ Descrição } & $\begin{array}{c}\text { Número de } \\
\text { empresas }\end{array}$ & $\begin{array}{c}\text { Número de } \\
\text { Observações }\end{array}$ \\
\hline Amostra Inicial - informações contábeis consolidadas na Economática ${ }^{\circledR}$ & 352 & 2.464 \\
Amostra após a exclusão das observações do ano de 2009 & 352 & 2.112 \\
Exclusão das companhias que não tinham dados disponíveis & 228 & 1.368 \\
Amostra Final & 124 & 744 \\
\hline
\end{tabular}

Fonte: Elaborado pelas autoras

No Tabela 3 é ilustrada a composição da amostra por dezoito setores de atividade estabelecidos na Economática ${ }^{\circledR}$. Os setores com maior participação no estudo foram Outros, Energia, Construção e Comércio com $17,7 \%, 16,1 \%, 9,7 \%$ e 8,9\%, respectivamente. O setor Outros é composto por empresas de vários ramos de atividade como, por exemplo, empresas de administração e empreendimentos, transporte ferroviário e educação. 
Tabela 3. Composição da Amostra por Setor

\begin{tabular}{|c|c|c|}
\hline Setor & $\begin{array}{l}\text { Número de } \\
\text { Observações }\end{array}$ & $\%$ \\
\hline Alimentos e Bebidas & 42 & $5.6 \%$ \\
\hline Comércio & 66 & $8.9 \%$ \\
\hline Construção & 72 & $9.7 \%$ \\
\hline Eletroeletrônicos & 6 & $0.8 \%$ \\
\hline Energia Elétrica & 120 & $16.1 \%$ \\
\hline Máquinas Industriais & 18 & $2.4 \%$ \\
\hline Mineração & 6 & $0.8 \%$ \\
\hline Minerais não Metálicos & 12 & $1.6 \%$ \\
\hline Outros & 132 & $17.7 \%$ \\
\hline Papel e Celulose & 18 & $2.4 \%$ \\
\hline Petróleo e Gás & 12 & $1.6 \%$ \\
\hline Química & 18 & $2.4 \%$ \\
\hline Siderurgia \& Metalurgia & 48 & $6.5 \%$ \\
\hline Software e Dados & 12 & $1.6 \%$ \\
\hline Telecomunicações & 12 & $1.6 \%$ \\
\hline Têxtil & 54 & $7.3 \%$ \\
\hline Transporte Serviços & 54 & $7.3 \%$ \\
\hline Veículos e peças & 42 & $5.6 \%$ \\
\hline Total & 744 & $100 \%$ \\
\hline
\end{tabular}

Fonte: Elaborado pelas autoras

Os modelos apresentados nas Equações 1 e 2 serão utilizados para identificar os determinantes da BTD. Esses modelos foram propostos por Koubaa e Anis (2015), sendo adaptados para esta pesquisa por meio da retirada da variável 'Ações institucionais', uma vez que essa variável não estava disponível nas demonstrações publicadas pelas companhias e não apresentou resultado significativo no estudo desses autores. Além disso, foram incluídas as variáveis de controle 'SETOR' e 'ANO'. As normas tributárias e algumas normas societárias podem ser diferentes em alguns setores, o que pode afetar a BTD. A adoção das IFRS no Brasil, de maneira completa, ocorreu no ano de 2010, porém a adoção prática pode ocorrer ao longo do tempo devido à curva de aprendizagem.

$$
\begin{aligned}
& D_{i t}=\beta_{0}+\beta_{1} R E N T+\beta_{2} R E V_{i t}+\beta_{3} I M O B_{i t}+\beta_{4} A D_{i t}+\beta_{5} I P L_{i t}+\beta_{6} L I Q_{i t}+\beta_{7} A L A V_{i t}+\beta_{8} T A M_{i t} \\
& +\beta_{9} A N O+\beta_{10} \text { SETOR }+\varepsilon \\
& D_{i t}=\beta_{0}+\beta_{1} R E N T+\beta_{2} R E V_{i t}+\beta_{3} I M O B_{i t}+\beta_{4} A D_{i t}+\beta_{5} I P L_{i t}+\beta_{6} L I Q_{i t}+\beta_{7} A L A V_{i t}+\beta_{8} T A M_{i t} \\
& +\beta_{9} \text { ANO }+\beta_{10} \text { SETOR }+\beta_{11} \text { LAGBTD }_{i t}+\varepsilon
\end{aligned}
$$

As Equações 1 e 2 serão testadas três vezes, sendo uma para cada tipo de BTD: total (BTD), temporária (BTDT) e permanente (BTDP). A descrição das variáveis é apresentada na Tabela 4. 
Tabela 4. Descrição das variáveis

\begin{tabular}{|c|c|c|}
\hline Variável & Fórmula & Descrição \\
\hline$B T D$ & $\mathrm{BTD}_{\mathrm{it}}=\frac{\left(\mathrm{LAIR}_{\mathrm{it}}-\mathrm{LT}_{\mathrm{it}}\right)}{\mathrm{AT}_{\mathrm{it}-1}}$ & $\begin{array}{l}\text { BDT é a diferença total entre o lucro contábil antes do imposto de renda } \\
\text { (LAIR) e o lucro tributário (LT); AT é ativo total defasado. O LT é } \\
\text { resultado da divisão da despesa de imposto de renda pela alíquota fiscal } \\
\text { máxima (34\%). }\end{array}$ \\
\hline$R E N T$ & & $\begin{array}{l}\text { RENT é uma variável binária com valor } 1 \text { quando o LAIR é positivo, e } \\
0 \text { caso contrário; }\end{array}$ \\
\hline$R E V$ & $\mathrm{REV}=\frac{\mathrm{REC}_{\mathrm{it}}-\mathrm{REC}_{\mathrm{it}-1}}{\mathrm{AT}_{\mathrm{it}-1}}$ & $\begin{array}{l}\text { REV é a variação da receita de vendas líquida (REC) das empresas } \\
\text { entre os anos } \mathrm{t}-1 \text { e t; dividida pelo AT defasado. }\end{array}$ \\
\hline$I M O B$ & $\mathrm{IMOB}_{\mathrm{it}}=\frac{\mathrm{IMOB}_{\mathrm{it}}-\mathrm{IMOB}_{\mathrm{it}-1}}{\mathrm{AT}_{\mathrm{it}-1}}$ & $\begin{array}{l}\text { IMOB é a variação do imobilizado bruto entre os anos } \mathrm{t}-1 \text { e } \mathrm{t} \text {; dividido } \\
\text { pelo AT defasado; }\end{array}$ \\
\hline$A D$ & $\mathrm{AD}=\frac{\text { LAIRit- } \mathrm{CxOP}}{\mathrm{AT}_{\mathrm{it}-1}}$ & $\begin{array}{l}\text { AD é o LAIR menos o fluxo de caixa das operações (CxOP) dividido } \\
\text { pelo ativo total defasado. }\end{array}$ \\
\hline$I P L$ & $\operatorname{IPL}_{\mathrm{it}}=\frac{\operatorname{COTAÇÃO}_{i t}}{\operatorname{LPA}_{\mathrm{it}}}$ & $\begin{array}{l}\text { IPL é a cotação da ação dividida pelo lucro por ação (LPA) no ano t; } \\
\text { COTAÇÃO é o preço de fechamento das ações. }\end{array}$ \\
\hline$L I Q$ & $\mathrm{LIQ}_{\mathrm{it}}=\frac{\mathrm{AC}_{\mathrm{it}}}{\mathrm{PC}_{\mathrm{it}}}$ & $\begin{array}{l}\text { LIQ é a relação entre o ativo circulante (AC) e o passivo circulante (PC) } \\
\text { da empresa i no ano t. }\end{array}$ \\
\hline$A L A V$ & $\frac{(\mathrm{LL}+\mathrm{PA}) * \mathrm{AT} /(\mathrm{PL}+\mathrm{PA})}{(\mathrm{LL}+\mathrm{PA}-\mathrm{RF})}$ & $\begin{array}{l}\text { ALA é a alavancagem financeira das empresas é lucro líquido (LL); } \\
\text { somado a participação acionária minoritária (PA); multiplicado pelo } \\
\text { AT; dividido pela soma do patrimônio líquido (PL) e a PA; dividido pela } \\
\text { soma do LL mais a PA, menos o resultado financeiro (RF). }\end{array}$ \\
\hline TAM & $\mathrm{LN}^{*}$ (ATit) & TAM é o logaritmo natural do AT. \\
\hline$L A G B T D$ & & LAGBTD $_{\text {it }}$ é a variável BTD defasada. \\
\hline$A N O$ & & Variável dummy para o período de estudo: 2009 a 2015. \\
\hline SETOR & & Variável dummy para os dezoito setores ilustrados na Tabela 3. \\
\hline$B T D T$ & $\mathrm{BTDT}_{\mathrm{it}}=\frac{\left(\mathrm{IRD}_{\mathrm{it}} / \mathrm{A}_{\mathrm{t}}\right)}{\mathrm{AT}_{\mathrm{it}-1}}$ & $\begin{array}{l}\text { BTDT é a BTD temporária; IRD é o imposto de renda diferido A é a } \\
\text { alíquota máxima de imposto de renda ( } 34 \%) \text {; dividida pelo AT. }\end{array}$ \\
\hline$B T D P$ & $\mathrm{BTDP}_{\mathrm{it}}=\mathrm{BTD}_{\mathrm{it}}-\mathrm{BTDT}_{\mathrm{it}}$ & BTDP é a BTD permanente. \\
\hline
\end{tabular}

Fonte: Elaborado pelas autoras.

As variáveis dependentes e independentes foram winsorizadas a 1\%, com o intuito de corrigir possíveis outliers, reduzindo o desvio-padrão das variáveis e a dispersão em torno da média (Vieira, Arruda, Lucena, \& Sena, 2015). Para efeito de comparação com os resultados de Koubaa e Anis (2015), a análise envolve regressão em Ordinary Least Squares (OLS) para dados em painel. Esses autores apresentaram os resultados utilizando pooled independente cross-sections (POLS), o que também será realizado neste estudo.

\section{ANÁLISE DOS RESULTADOS}

A Tabela 5 registra a estatística descritiva. Os resultados encontrados para a média da $B T D(0,004)$, da $B T D T(-0,004)$ e da $B T D P(0,008)$ estão próximos daqueles encontrados por Marques et al. (2016), em companhias abertas de cinco países da América Latina e por Costa e Lopes (2015) para o Brasil.

Tabela 5. Análise Descritiva

\begin{tabular}{lrrrrr}
\hline \multicolumn{1}{c}{ Variável } & Média & Desvio Padrão & Mínimo & Máximo & Mediana \\
\hline BTD & 0,004 & 0,073 & $-0,316$ & 0,228 & 0,006 \\
RENT & 0,780 & 0,415 & 0,000 & 1,000 & 1,000 \\
REV & 0,058 & 0,128 & $-0,367$ & 0,466 & 0,048
\end{tabular}


Tabela 5. Análise Descritiva (continuação)

\begin{tabular}{lrrrrr}
\hline \multicolumn{1}{c}{ Variável } & Média & Desvio Padrão & Mínimo & Máximo & Mediana \\
\hline IMOB & 0,001 & 0,104 & $-0,568$ & 0,252 & 0,003 \\
AD & $-0,055$ & 0,061 & $-0,330$ & 0,104 & $-0,051$ \\
IPL & 12,263 & 23,526 & $-58,194$ & 131,816 & 9,173 \\
LIQ & 1,846 & 1,114 & 0,302 & 6,930 & 1,650 \\
ALA & 1,289 & 7,244 & $-42,800$ & 28,200 & 1,700 \\
TAM & 15,212 & 1,509 & 12,475 & 19,559 & 15,163 \\
LAGBTD & 0,007 & 0,072 & $-0,316$ & 0,193 & 0,014 \\
BTDT & $-0,004$ & 0,038 & $-0,150$ & 0,131 & $-0,001$ \\
BTDP & 0,008 & 0,079 & $-0,316$ & 0,264 & 0,013 \\
\hline Fonte: Elaborada
\end{tabular}

Fonte: Elaborada pelas autoras.

Na Tabela 6, são apresentadas as correlações entre as variáveis da pesquisa. A variável BTD (Tabela 6) evidencia correlação positiva e significativa com a variável rentabilidade (RENT), variação da receita de vendas ( $R E V$ ), liquidez ( $L I Q)$ semelhante aos resultados de Koubaa e Anis (2015). O que já era esperado, conforme exposto na Tabela 1, é que empresas altamente rentáveis com variações nas receitas de vendas e elevados índices de liquidez, procuram se beneficiar das ambiguidades da legislação tributária, numa tentativa de reduzir a base tributária, para o pagamento de menores tributos, aumentando as BTD.

Tabela 6. Correlação de Pearson

\begin{tabular}{|c|c|c|c|c|c|c|c|c|c|c|c|c|}
\hline & BTD & RENT & REV & IMOB & AD & IPL & LIQ & ALA & TAM & LBTD & BTDT & BTDP \\
\hline BTD & 1,000 & & & & & & & & & & & \\
\hline \multirow[t]{2}{*}{ RENT } & 0,369 & 1 & & & & & & & & & & \\
\hline & 0,000 & & & & & & & & & & & \\
\hline \multirow[t]{2}{*}{ REV } & 0,099 & 0,205 & 1 & & & & & & & & & \\
\hline & 0,007 & 0,000 & & & & & & & & & & \\
\hline \multirow[t]{2}{*}{ IMOB } & $-0,029$ & 0,054 & 0,121 & 1 & & & & & & & & \\
\hline & 0,430 & 0,139 & 0,001 & & & & & & & & & \\
\hline \multirow[t]{2}{*}{ AD } & 0,257 & 0,425 & 0,156 & 0,040 & 1 & & & & & & & \\
\hline & 0,000 & 0,000 & 0,000 & 0,282 & & & & & & & & \\
\hline \multirow[t]{2}{*}{ IPL } & 0,072 & 0,373 & 0,030 & 0,066 & 0,059 & 1 & & & & & & \\
\hline & 0,049 & 0,000 & 0,414 & 0,073 & 0,107 & & & & & & & \\
\hline \multirow[t]{2}{*}{ LIQ } & 0,197 & 0,180 & $-0,022$ & 0,040 & 0,338 & 0,008 & 1,000 & & & & & \\
\hline & 0,000 & 0,000 & 0,550 & 0,271 & 0,000 & 0,836 & & & & & & \\
\hline \multirow[t]{2}{*}{ ALA } & 0,097 & 0,161 & 0,008 & 0,006 & 0,191 & 0,053 & 0,032 & 1 & & & & \\
\hline & 0,008 & 0,000 & 0,819 & 0,872 & 0,000 & 0,152 & 0,392 & & & & & \\
\hline \multirow[t]{2}{*}{ TAM } & 0,021 & 0,058 & 0,051 & $-0,003$ & $-0,120$ & 0,021 & $-0,253$ & $-0,018$ & 1 & & & \\
\hline & 0,564 & 0,115 & 0,165 & 0,946 & 0,001 & 0,562 & 0,000 & 0,617 & & & & \\
\hline \multirow[t]{2}{*}{ LBTD } & 0,475 & 0,653 & 0,241 & $-0,013$ & 0,614 & 0,157 & 0,286 & 0,157 & 0,076 & 1 & & \\
\hline & 0,000 & 0,000 & 0,000 & 0,732 & 0,000 & 0,000 & 0,000 & 0,000 & 0,039 & & & \\
\hline \multirow[t]{2}{*}{ BTDT } & 0,165 & 0,213 & $-0,041$ & $-0,017$ & 0,073 & 0,058 & 0,048 & 0,019 & 0,041 & 0,310 & 1 & \\
\hline & 0,000 & 0,000 & 0,266 & 0,645 & 0,048 & 0,117 & 0,195 & 0,599 & 0,261 & 0,000 & & \\
\hline \multirow[t]{2}{*}{ BTDP } & 0,850 & 0,236 & 0,117 & $-0,022$ & 0,204 & 0,040 & 0,165 & 0,078 & $-0,005$ & 0,288 & $-0,365$ & 1 \\
\hline & 0,000 & 0,000 & 0,001 & 0,550 & 0,000 & 0,279 & 0,000 & 0,033 & 0,898 & 0,000 & 0,000 & \\
\hline
\end{tabular}

Fonte: Elaborada pelas autoras. 
Os acrruals $(A D)$ apresentam correlação positiva e significativa com a variável $B T D$, ou seja, quanto maior os accruals, maiores são as BTD. Esse resultado sugere que a BTD pode ser utilizada para detectar práticas de gerenciamento de resultados, corroborando o estudo de Ferreira et al. (2012). A variável cotação das ações dividida pelo lucro por ação (IPL) também expõe correlação positiva e significativa com a $B T D$, ou seja, quanto maior a cotação das ações dividida pelo lucro por ação, maior a $B T D$. Esses resultados divergem dos encontrados por Koubaa e Anis (2015). A variável tamanho da empresa (TAM) não assinalou correlação significativa com a variável $B T D$, o que difere do resultado encontrado por Koubaa e Anis (2015) em empresas tunisianas.

A variável alavancagem (ALA) apresenta correlação positiva e significativa com a variável BTD, ou seja, quanto maior o nível de endividamento, maior a BTD. Esse resultado sugere que as empresas alavancadas aumentam as incertezas dos investidores, e os elevados níveis de endividamentos sinalizam maior risco de mercado. A variável $L G B T D$ foi usada como uma variável de controle para indicar o efeito da BTD no tempo t-1 sobre a $B T D$ no tempo t. Destaca-se o fato de a variável $L A G B T D$ (BTD defasada) apresentar correlação positiva e significativa com a variável $B T D$, ou seja, quanto maior a $B T D$ do ano anterior, maior a $B D T$, corroborando os estudos de Koubaa e Anis (2015).

Na Tabela 7 são registrados os resultados da regressão para o modelo especificado na Equação 1 e Equação 2, com a variável dependente $B T D$ total.

Tabela 7. Fatores Determinantes da BTD Total

\begin{tabular}{lcccccc}
\hline \multicolumn{1}{c}{ Variável } & \multicolumn{3}{c}{ Equação 1 } & \multicolumn{3}{c}{ Equação 2 } \\
\hline Fatores Institucionais & Coeficiente & $\boldsymbol{t}$ & $\boldsymbol{p}$-valor & Coeficiente & $\boldsymbol{t}$ & $\boldsymbol{p}$-valor \\
RENT & 0,058 & 7,46 & $0,000^{* * *}$ & 0,024 & 2,57 & $0,010^{* *}$ \\
REV & 0,021 & 0,87 & 0,385 & 0,001 & 0,04 & 0,968 \\
IMOB & $-0,037$ & $-1,92$ & $0,055^{*}$ & $-0,021$ & $-1,10$ & 0,271 \\
Fatores Não Institucionais & & & & & & \\
AD & 0,083 & 1,20 & 0,230 & $-0,105$ & $-1,48$ & 0,140 \\
IPL & 0,000 & $-1,08$ & 0,279 & 0,000 & $-0,81$ & 0,416 \\
LIQ & 0,009 & 3,30 & $0,001^{* * *}$ & 0,006 & 2,24 & $0,025^{* *}$ \\
Variáveis de Controle & & & & & & \\
ALA & 0,000 & 0,41 & 0,682 & 0,000 & 0,36 & 0,718 \\
TAM & 0,001 & 0,62 & 0,532 & $-0,001$ & $-0,65$ & 0,516 \\
ANO & 0,002 & 1,56 & 0,119 & 0,003 & 2,05 & $0,040^{* *}$ \\
SETOR & $-0,001$ & $-2,33$ & $0,020^{* *}$ & $-0,001$ & $-1,87$ & $0,062^{*}$ \\
LAGBTD & & & & 0,429 & 6,40 & $0,000^{* * *}$ \\
Constante & $-4,615$ & $-1,58$ & 0,114 & $-5,851$ & $-2,06$ & $0,040^{* *}$ \\
No. OBS & 744 & & & 744 & & \\
F (prob) & 11,02 & $(0,000)$ & & 11,81 & & $(0,000)$ \\
R-squared & 0,176 & & & 0,249 & & \\
Root MSE & 0,066 & & & 0,063 & & \\
\hline
\end{tabular}

Nota: ***,** e* denotam a significância estatística nos níveis de $1 \%, 5 \%$ e $10 \%$, respectivamente.

Fonte: Elaborada pelas autoras.

A estatística $\mathrm{F}$ significante a $1 \%$ sugere adequação do modelo. Os modelos testados nesta pesquisa não apresentaram estatísticas VIF elevadas para nenhuma variável explicativa (todas inferiores a 2,49), o que sugere inexistência de multicolinearidade dessas variáveis. O poder explicativo das variáveis independentes é maior na Equação 2 (R-squared de 0,249), sugerindo que a inclusão da variável $L G B T D$ é adequada.

Os coeficientes positivos e significativos (ao nível de 1\% na Equação 1 e 5\% na Equação 2) das variáveis $R E N T$ e $L I Q$ sugerem que a rentabilidade e a liquidez têm relação direta com a BTD. Os resultados corroboram os 
estudos de Manzon e Plesko (2002) e Koubaa e Anis (2015). Esses resultados insinuam que empresas altamente rentáveis e com alta liquidez procuram reduzir a base tributária, numa tentativa de reduzir os tributos a serem pagos, aumentando a $B T D$. Esses dados confirmam as hipóteses H1 e H6 desta pesquisa, de que a rentabilidade e a liquidez, respectivamente, afetam positivamente a $B T D$.

Por outro lado, o coeficiente negativo $(-0,037)$ e significativo (ao nível de $10 \%$ ) da variável IMOB na Equação 1 sugere que quanto menor o nível de imobilização, maior o valor da BTD. Contrariando os resultados encontrados por Manzon e Plesko (2002), que encontraram relação positiva e significativa entre a variável IMOB e a BTD. Sendo assim, não se confirma a hipótese $\mathrm{H}_{3}$ estabelecida na presente pesquisa. Essa variável, na Equação 2 , não revela significância em relação à variável $B T D$, resultado que confirma o que foi encontrado por Koubaa e Anis (2015). Os resultados divergentes na Equação 1 e 2, quanto à variável $I M O B$, podem sugerir que esta variável pode não ser adequada para a análise da $B T D$ no Brasil. Isto pode ser decorrente do fato de a maioria das empresas brasileiras ainda utilizar, após a adoção das IFRS, os mesmos métodos de depreciação de antes dessa adoção (Telles, \& Salotti, 2015). O coeficiente positivo e significativo (ao nível de 1\%) da variável LAGBTD $(0,429)$, aponta que quanto maior a $B T D$ do ano anterior, maior a $B T D$ do ano seguinte. Esse resultado sugere que os gestores podem observar o valor da $B T D$, quando da preparação das demonstrações contábeis.

As variáveis crescimento das vendas (REV), acrruals $(A D)$, a cotação da ação dividida pelo lucro por ação (IPL), alavancagem (ALA) e tamanho da empresa (TAM), não apresentaram valores significativos nesta pesquisa, ou seja, não afetam a variável BTD. Sendo assim, não confirmando as hipóteses H2, H4, H5, H7 e H8, estabelecidas no trabalho. Contrariando os resultados encontrados por Koubaa e Anis (2015), que encontraram relação positiva e significativa com as variáveis $R E V, A D$ e $A L A$ e relação significativa e negativa com a variável $I P L$.O setor de atividade parece ser relevante na análise da $B T D$, uma vez que o coeficiente da variável SETOR é significativo na Equação 1 e 2, sugerindo que a $B T D$ pode variar entre os setores de atividade. A variável $A N O$ apresentou coeficiente significativo (ao nível de 10\%) apenas na Equação 2, não permitindo inferências sobre essa variável.

Na Tabela 8 são apresentados os resultados para a s Equações 1 e 2 considerando a variável dependente $B T D$ temporária $(B T D T)$.

Tabela 8. Fatores Determinantes da BTD Temporária (BTDT)

\begin{tabular}{|c|c|c|c|c|c|c|}
\hline \multirow[b]{2}{*}{ Variável } & \multicolumn{3}{|c|}{ Equação 1} & \multicolumn{3}{|c|}{ Equação 2} \\
\hline & Coeficiente & $t$ & p-valor & Coeficiente & $t$ & p-valor \\
\hline \multicolumn{7}{|l|}{ Fatores Institucionais } \\
\hline RENT & 0,022 & 4,67 & $0,000 * * *$ & 0,019 & 3,90 & $0,000 * * *$ \\
\hline$R E V$ & $-0,024$ & $-1,57$ & 0,116 & $-0,025$ & $-1,39$ & 0,165 \\
\hline$I M O B$ & $-0,005$ & $-0,35$ & 0,727 & 0,040 & 1,02 & 0,309 \\
\hline \multicolumn{7}{|c|}{ Fatores Não Institucionais } \\
\hline$A D$ & $-0,005$ & $-0,12$ & 0,903 & 0,005 & 0,13 & 0,899 \\
\hline$I P L$ & 0,000 & $-0,88$ & 0,381 & 0,000 & $-0,78$ & 0,438 \\
\hline$L I Q$ & 0,001 & 0,44 & 0,657 & $-0,001$ & $-0,37$ & 0,709 \\
\hline \multicolumn{7}{|l|}{ Variáveis de Controle } \\
\hline$A L A$ & 0,000 & $-0,39$ & 0,700 & 0,000 & $-0,48$ & 0,629 \\
\hline TAM & 0,001 & 0,92 & 0,357 & 0,000 & $-0,06$ & 0,952 \\
\hline$A N O$ & 0,000 & $-0,18$ & 0,858 & 0,000 & 0,09 & 0,927 \\
\hline SETOR & 0,000 & $-0,17$ & 0,869 & 0,000 & $-0,40$ & 0,690 \\
\hline$L A G B T D T$ & & & & 0,112 & 2,00 & $0,046^{* *}$ \\
\hline Constante & 0,290 & 0,16 & 0,872 & $-0,220$ & $-0,10$ & 0,923 \\
\hline No. OBS & 744 & & & 744 & & \\
\hline $\mathrm{F}$ (prob) & 2,97 & $(0,000)$ & & 2,50 & & $(0,000)$ \\
\hline R-squared & 0,054 & & & 0,082 & & \\
\hline Root MSE & 0,037 & & & 0,037 & & \\
\hline
\end{tabular}

Nota: $* * *, * * \mathrm{e} *$ denotam a significância estatística nos níveis de $1 \%, 5 \%$ e $10 \%$, respectivamente.

Fonte: Elaborada pelas autoras.

Quando a variável dependente é a BTDT, apenas as varáveis rentabilidade e (RENT) e BTDT defasada 
(LGBTDT) apresentaram coeficiente significativo. O coeficiente positivo e significativo (ao nível de $1 \%$ ) entre a variável rentabilidade $(R E N T)$ e a $B T D T$ mostra que quanto maior a rentabilidade da empresa, maior o nível de $B T D T$. Dessa forma, aceita-se a hipótese H1, de que quanto maior a rentabilidade, maior o nível de BTDT. Ressalta-se o fato de haver coeficiente positivo e significativo (ao nível de 5\%) entre a BTDT e a variável defasada $\angle A G B D T$, ou seja, quanto maior a BTDT do ano anterior, maior a BTDT do ano.

Na Tabela 9, estão expostos os resultados das equações 1 e 2 para identificar os determinantes da $B T D$ permanente $(B T D P)$.

Tabela 9. Fatores Determinantes da BTD Permanente (BTDP)

\begin{tabular}{|c|c|c|c|c|c|c|}
\hline \multirow[b]{2}{*}{ Variável } & \multicolumn{3}{|c|}{ Equação 1} & \multicolumn{3}{|c|}{ Equação 2} \\
\hline & Coeficiente & $t$ & p-valor & Coeficiente & $t$ & p-valor \\
\hline \multicolumn{7}{|l|}{ Fatores Institucionais } \\
\hline RENT & 0,035 & 3,75 & $0,000 * * *$ & 0,036 & 2,95 & $0,003 * * *$ \\
\hline$R E V$ & 0,049 & 1,56 & 0,118 & 0,055 & 1,50 & 0,135 \\
\hline$I M O B$ & $-0,035$ & $-1,47$ & 0,141 & $-0,060$ & $-0,97$ & 0,331 \\
\hline \multicolumn{7}{|c|}{ Fatores Não Institucionais } \\
\hline$A D$ & 0,087 & 1,07 & 0,285 & 0,110 & 1,27 & 0,203 \\
\hline$I P L$ & 0,000 & $-0,61$ & 0,543 & 0,000 & $-0,97$ & 0,331 \\
\hline$L I Q$ & 0,009 & 2,97 & $0,003 * * *$ & 0,008 & 2,52 & $0,012 * *$ \\
\hline \multicolumn{7}{|l|}{ Variáveis de Controle } \\
\hline$A L A$ & 0,000 & 0,46 & 0,642 & 0,000 & 0,47 & 0,640 \\
\hline TAM & 0,000 & 0,04 & 0,968 & 0,001 & 0,26 & 0,793 \\
\hline$A N O$ & 0,002 & 1,18 & 0,237 & 0,002 & 0,80 & 0,426 \\
\hline SETOR & $-0,001$ & $-2,13$ & $0,033 * *$ & $-0,001$ & $-1,72$ & $0,086^{*}$ \\
\hline$\angle A G B T D P$ & & & & $-0,018$ & $-0,20$ & 0,845 \\
\hline Constante & $-4,102$ & $-1,19$ & 0,234 & $-3,632$ & $-0,81$ & 0,421 \\
\hline No. OBS & 744 & & & 744 & & \\
\hline $\mathrm{F}$ (prob) & 4,31 & $(0,000)$ & & 3,36 & & 00) \\
\hline R-squared & 0,091 & & & 0,094 & & \\
\hline Root MSE & 0,075 & & & 0,078 & & \\
\hline
\end{tabular}

Nota: ***,** e* denotam a significância estatística nos níveis de $1 \%, 5 \%$ e $10 \%$, respectivamente.

Fonte: Elaborada pelas autoras.

A rentabilidade está, positivamente, associada com a $B T D P$, com a BTDT e a $B T D$ total, ou seja, quanto maior a rentabilidade, maior o nível de $B T D$. Esses resultados são condizentes com os resultados encontrados por Koubaa e Anis (2015) e Manzon e Plesko (2002) para a BTD total, de que as empresas rentáveis buscam a redução da carga tributária para pagamento de menores impostos.

A variável liquidez $(L I Q)$ está positivamente associada com o nível de BTD permanente, ou seja, quanto maior a liquidez das empresas, maior o nível de $B T D$ permanente. Nota-se que a variável $L A G B T D P$ não registrou valores significativos, ou seja, a $B T D$ do ano anterior não influencia na $B T D$ permanente do ano.

Os resultados apresentados sugerem que separar a BTD por tipos permanente e temporária é relevante para identificação dos fatores determinantes da $B T D$. Além disso, o setor de atividade parece ser uma variável de controle relevante nessa análise. 


\section{CONSIDERAÇÕES FINAIS}

O objetivo deste trabalho é identificar quais são os fatores institucionais e não institucionais que determinam as book-tax differences (BTD) das companhias abertas brasileiras. A amostra da pesquisa é composta por 124 companhias com dados disponíveis para o período de 2010 a 2015 . Os resultados da pesquisa sugerem que a BTD total apresenta coeficiente positivo e significativos com as variáveis rentabilidade, liquidez e a BTD defasada. Quando desmembrados os tipos de BTD (temporária e permanente), a rentabilidade, e a BTDT defasada estão positivamente associadas à BTD temporária. A rentabilidade e a liquidez estão positivamente associadas à BTD permanente. Logo, sugere-se que quanto maior o nível dessas variáveis, maiores os tipos de BTD.

A rentabilidade (fator institucional) está associada com todos os tipos de BTD (total, temporária e permanente). Esta é a variável que parece implicar mais no aumento da BTD, corroborando o estudo de Koubaa e Anis (2015) para quem empresas altamente rentáveis, geralmente, aproveitam as ambiguidades da legislação tributária para reduzir o pagamento de impostos, aumentando a BTD. Diferentemente dos resultados encontrados por Koubaa e Anis (2015) para uma amostra de companhias de um país common law, as variáveis crescimento das vendas, accruals e retorno das ações não apresentaram coeficientes significativos. Esses resultados são inesperados, uma vez que seria esperado maior gerenciamento de resultados no Brasil devido à origem code law.

A abordagem de pesquisa é considerada inovadora, principalmente no contexto brasileiro, pois são poucos os estudos que buscam identificar os fatores que determinam a BTD, separando-os em institucionais e não institucionais. O trabalho contribui com a disseminação do conhecimento sobre o assunto e com o possível esclarecimento de questionamentos a respeito dos fatores que determinam a BTD em companhias abertas brasileiras. A quantidade limitada de pesquisas anteriores sobre o tema desta pesquisa pode se configurar como uma limitação para o embasamento teórico, delimitando esta pesquisa como exploratória. Para pesquisas futuras, sugere-se aumentar a amostra utilizada, comparando-a com a de outros países code law e common law.

\section{REFERÊNCIAS}

Chan, K.H., Lin, K. Z., \& Mo, P. L.L (2010). Will a departure from tax-based accounting encourage tax noncompliance? Archival evidence from a transition economy. Journal of Accounting and Economics, 50(1), $58-73$

Costa, P. S., \& Lopes, A. B. (2015). Implicações da adoção das IFRS sobre as book-tax differences: o caso do Brasil. São Paulo: Novas Edições Acadêmicas.

Desai, M. A (2005). The degradation of reported corporate profits. Journal of Economics Perspectives, 19(4), 171-192.

Frank, M. M., Lynch, L. J., \& Rego, S. O. (2009). Tax reporting aggressiveness and its relation to aggressive financial reporting. The Accounting Review, 84(2), 467-496.

Ferreira, F. R., Matinez, A. L., Costa, F. M., \& Passamani, R. R. (2012). Book-tax differences e gerenciamento de resultados no mercado de ações do Brasil. RAE-Revista de Administração de Empresas, 52(5), 488-501.

Formigoni, H, Antunes, M.T. P., \& Paulo, E. (2009). Diferença entre o lucro contábil e lucro tributável: uma análise sobre o gerenciamento de resultados contábeis e gerenciamento tributário nas companhias abertas brasileiras. BBR Brazilian Business Review, 6(1), 44-61.

Hanlon, M. (2005). The persistence and pricing of earnings, accruals and cash flows when firms have large booktax differences. The Accounting Review, 80(1), 137-166.

Koubaa, R. R., \& Anis, J. (2015). Book-tax differences: relevant explanatory factors. International Journal of Accounting and Economics Studies, 3(2) 95-104.

Kvaal, E., \& Nobes, C. (2013). The Development of Book-Tax Differences in Seven Major Countries. Recuperado em: http://ssrn.com/abstract=2391780.

Long, Y., Ye, K., \& Lv, M. (2013). Non-institutional Determinants of Book-Tax Differences: evidence from China. Journal of Accounting and Finance, v. 13, n. 3, p. 146. 
Manzon, G. B, \& Plesko, G. (2002).The relation between financial and tax reporting measures of income. Tax Law Review, 55(1), 175-214.

Marques, A.V. C., Costa, P. S., \& Silva, P. R. (2016). Relevância do Conteúdo Informacional das Book-Tax Differences para Previsão de Resultados Futuros: Evidências de Países-Membros da América Latina. Revista Contabilidade \& Finanças, 27(70), 29-42.

Mills, L. F., Newberry, K.J., \& Trautman, W. B (2002). Trends in book-tax income and balance sheet differences. Recuperado em: https://ssrn.com/abstract=313040.

Mills, L. F., \& Newberry, K.J. (2001). The Influence of Tax and Nontax Costs on Book-Tax Reporting Differences: public and private firms. The Journal of the American Taxation Association: Spring, 23(1), 1-19.

Nakao, S. H. (2015). A adoção de IFRS e o legado da conformidade contábil- fiscal mandatória. Tese de livre docência, Faculdade de Economia, Administração e Contabilidade, Universidade de São Paulo, Ribeirão Preto, SP, Brasil.

Niyama, J. K., \& Silva, C. A. T.(2011). Teoria da Contabilidade. 2a ed. São Paulo: Atlas.

Plesko, G. A (2004). Corporate tax avoidance and the properties of corporate e earnings. National Tax Journal, 729-737.

Sodan, S. (2012). Book-tax differences and companies financial characteristics: the case of Croatia. The Business Review Cambridge, 19(2), 265-271.

Tang, T. (2006). Book-tax differences: a function of accounting-tax misalignment, earnings management and tax management: empirical evidence from China. In: American Accounting Association Annual Meeting, 2006, Washington.

Tang, T. \& Firth, M (2011). Can Book-Tax Differences Capture Earnings Management and Tax Management? Empirical Evidence from China. The International Journal of Accounting, 46(2), 175-204.

Telles, S.V., \& Salotti, B. M. (2015). Divulgação da informação contábil sobre depreciação: o antes e o depois da adoção das IFRS. Revista Universo Contábil, 11(2), 153-173.

Vieira, C. A. M., Arruda, M. P., Lucena, W. G. L., \& Sena, E.W.S. (2015). Análise do impacto do grau de endividamento e da diversificação das atividades sobre o conservadorismo contábil. In: Congresso Anpcont. Anais...Curitiba. Paraná, 0915. 